\title{
Analysis of Features Contributing to Activity of the Single- Stranded Origin of Bacillus Plasmid pBAA1
}

\author{
LIAM SEERY AND KEVIN M. DEVINE* \\ Department of Genetics, Trinity College, Dublin 2, Ireland
}

Received 27 July 1992/Accepted 29 January 1993

\begin{abstract}
The features which contribute to the activity of the single-stranded origin of the Bacillus plasmid pBAA1 were investigated. This origin is contained on a DNA fragment greater than 116 but less than 191 bases in size. There is the potential to form three stem-loop structures within this fragment. Comparison of the sequence of this origin from pBAA1 with the sequence of a homologous fragment from the Bacillus thuringiensis plasmid pGI2 indicates that both the structure and the relative positioning of the predicted stem-loops are important for origin activity. Deletion analysis suggests that it is the structure of stem-loop III which is important, because it can be replaced by a nonrelated dyad element without significant loss of origin activity. Three sequence motifs are conserved between the origins from pBAA1 and pGI2. Mutation of motif 1 leads to attenuation of single-stranded origin activity. A second motif (motif 3 ) shares significant homology with a group of single-strand initiation (ssi) sites found on plasmids isolated from Escherichia coli, suggesting that it also contributes to single-stranded origin activity. Our results also indicate that RNA polymerase is utilized to synthesize the RNA primer at the pBAA1 single-stranded origin and that this origin can function in both Bacillus subtilis and Staphylococcus aureus.
\end{abstract}

The inability of DNA polymerases to synthesize DNA de novo and in a $3^{\prime}-5^{\prime}$ direction has prompted investigation of the mechanism whereby DNA lagging-strand synthesis is initiated. The single-stranded DNA phages, which are converted from a single-stranded to a double-stranded form during their replication cycle, are an excellent model system to investigate this problem (16). Upon entry into the cell, the single-stranded phage genome is coated with single-stranded DNA binding protein leaving a region of secondary structure exposed. These exposed stem-loop structures function as origins of replication (in phages called minus origins) for the conversion of single-stranded phage to the double-stranded form. Three pathways of initiation of second-strand synthesis have been distinguished on the basis of the minus origin sequence and host function requirements. These are represented by the minus origins found on the phages $\mathrm{G} 4, \mathrm{M} 13$, and $\phi X 174$ (see references 4 and 5, for reviews). In each case, an RNA primer is synthesized to initiate DNA synthesis. This RNA primer is synthesized by DNA primase in the case of G4 and by RNA polymerase in the case of M13. Conversion of $\phi \times 174$ to the double-stranded form in contrast requires the formation of a primosome, a multiprotein complex composed of at least seven host proteins (DnaB, DnaC, DnaG, factor $T(i), n, n^{\prime}$, and $\left.n^{\prime \prime}\right)(2,3)$. The primosome assembles at a primosome assembly site and can translocate in both directions along the single-stranded DNA template synthesizing RNA primers at intervals (18). It can thus be viewed as a mobile replication promoter which synthesizes RNA primers along the DNA template to initiate lagging-strand synthesis.

In addition to the minus origins of the single-stranded phages, a number of sequences on the Escherichia coli chromosome (33) and on plasmids isolated from $E$. coli (21, 22) at which lagging-strand DNA synthesis is initiated have been identified. These single-strand initiation sequences (ssi) were identified by their ability to act as sites for initiation of

\footnotetext{
* Corresponding author.
}

complementary strand synthesis of an M13 phage derivative which had its own minus origin deleted $(15,21,22,26)$. These ssi sites fall into a number of groups on the basis of sequence homology $(21,22)$. Group I [which includes $\operatorname{ssi} A(\mathrm{~F}-\mathrm{f} 5)$, ssiA(ColE2), and $s s i A(\mathrm{ColE} 1)]$ and group II [which includes $s s i C(\mathrm{~F}-2 \mathrm{~b})$ and $s s i B(\mathrm{R} 100)$ ] sites are approximately 100 nucleotides in length, have the potential to form secondary structure, have a high degree of sequence homology within each group, and probably function by a $\phi X 174-$ type mechanism (21). Group III sites, which include ssiE(Ff6), ssiB(R6K), and ssiA(R100), are greater than 200 nucleotides in length, have conserved sequence motifs different from the other two groups, and probably function by a G4-type mechanism. A novel pathway, termed the ABC primosome, has been identified for $\operatorname{ssi} A(\mathrm{R} 6 \mathrm{~K})$ which functions with a primosome composed of DnaA, DnaB, DnaC, and DNA primase (20). Only group I ssi sites show sequence similarity to the minus origin of $\phi X 174$ (21). Thus, it is evident that there are a number of different pathways in $E$. coli whereby lagging-strand synthesis can be initiated. A group of plasmids have been isolated, primarily from grampositive organisms, which replicate by the rolling circle-type mechanism typical of the single-stranded coliphages (for reviews, see references 9 and 23). These plasmids (termed rolling circle plasmids) also contain sequences which are involved in the conversion of the single-stranded replication intermediate to the double-stranded plasmid form. For the plasmids, these elements are termed single-stranded origins (SSOs). Among the rolling circle group of plasmids, three classes of SSO have been identified, represented by those found on pT181, pUB110, and pBAA1, respectively. They can be distinguished by sequence, host range, and the effect of their deletion on plasmid copy number and segregational stability. The three classes of SSO have little sequence homology, but each has the potential to form secondary structure. Only the SSO of pUB110 has been reported to function in a host other than its natural one (6). Deletion of the SSO can lead to accumulation of single-stranded plasmid DNA, a decrease in copy number, and an increase in the 
segregational instability of the plasmid. These effects depend on both the plasmid and the host in which it is resident $(6,7$, $10,35)$. All three phenotypes are probably caused by inefficient conversion of the plasmid from the single-stranded to the double-stranded form. Thus, it is evident that, like in $E$. coli, there are a number of different pathways whereby lagging-strand synthesis can be initiated in gram-positive organisms.

SSOs of replication use host functions to initiate secondstrand synthesis. Thus, the number and type of such origins which function in a particular host will reflect the mechanisms used by the cell to initiate chromosomal lagging-strand synthesis. In view of this, we chose to investigate the SSO of pBAA1 to determine which features are important for activity. We show that the pBAA1 SSO is similar in size to those identified in gram-negative organisms. There is the potential within this sequence to form three stem-loop structures. The predicted stem-loop III can be replaced by a dyad element of little sequence similarity without significant loss of origin activity, suggesting that the structure of stem-loop III is an important feature of the SSO. Three sequence motifs are conserved between the SSOs of pBAA1 and pGI2 (isolated from Bacillus thuringiensis), suggesting that they contribute to origin activity. Mutation of motif 1 leads to attenuation of origin activity. A second motif (motif 3 ) is also found among a group of $s s i$ sites isolated from plasmids resident in $E$. coli.

\section{MATERIALS AND METHODS}

Bacteria and plasmids. E. coli $\mathrm{Tg} 1$ (Amersham) and Bacillus subtilis JH642 were used throughout this study. The plasmids used were pRP22 (7); pRP22 2 PstI, which has the PstI site in the multiple cloning site of pRP22 deleted and was used to generate deletions in the SSO either from the unique PstI site (pRP/P series) or XmaIII (pRP/X series) site by using Bal 31; pC194 $\triangle \mathrm{MO}$, a kind gift from A. Gruss (INRA, Jouy-en-Josas, France), which has had the palA sequence deleted; and a HindIII-NaeI fragment from pRP22, which contains the SSO, and was cloned into the PvuII site of $\mathrm{pC} 194 / \Delta \mathrm{MO}$ in both orientations.

DNA manipulations. Restriction enzymes were obtained commercially and used as directed by the supplier. All DNA manipulations were carried out according to the method of Sambrook et al. (28). Single-stranded plasmid DNA was detected by the method of te Riele et al. (34) with the modifications described by Devine et al. (7). Sequencing was carried out by the dideoxy chain termination method of Sanger et al. (29) with double-stranded plasmid templates. Sequencing kits were obtained from Promega Corporation (Madison, Wis.), and reactions were carried out with either universal primers or oligonucleotides synthesized on an Applied Biosystems model 391 PCR Mate. The rifampin sensitivity of conversion from the single-stranded to the double-stranded plasmid forms was tested by the method of Boe et al. (6).

Plasmid transformation. Competent $E$. coli cells were made and transformed according to the method of Sambrook et al. (28). Staphylococcus aureus protoplasts were prepared for transformation according to the method of O'Reilly and Foster (25). B. subtilis JH642 was transformed according to the method of Anagnostopoulos and Spizizen (1).

Computer analysis. Sequences similar to the pBAA1 SSO were obtained by homology searches of the GenBankEMBL DNA sequence data library with similarity search software related to the ACNUC retrieval system (13). Se-

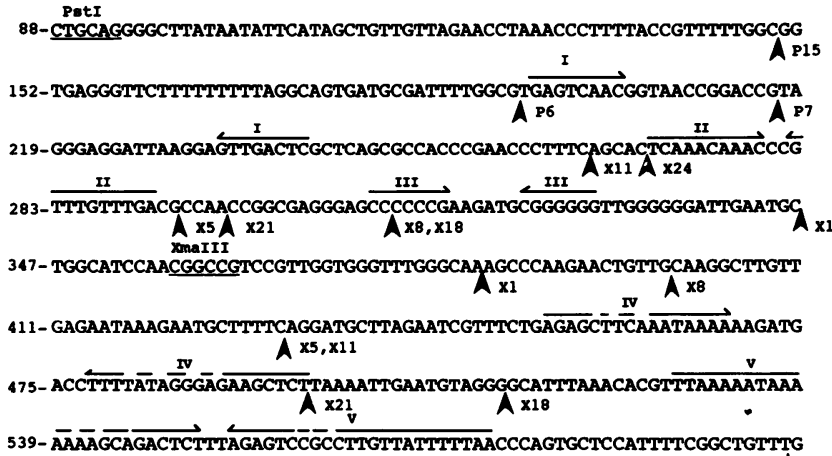

603-GAAAT

FIG. 1. Sequence of the SSO-containing fragment of the plasmid pBAA1. The numbering of the sequence is the same as in Devine et al. (7). This origin is contained on the PstI-XmaIII fragment which has the potential to form three stem-loop structures (stem-loops are indicated by roman numerals $I$ through $V$ placed over the inverted repeat half-arrows). The sequences of predicted stem-loops IV and $\mathrm{V}$, which are located outside the SSO, are also shown. Inverted repeats are indicated by half-arrows over the sequence. The deletion endpoints in the plasmid constructs are indicated by arrowheads under the sequence with the name of the deletion beside them. The $X$ series of deletions have two endpoints because of the bidirectional nature of $\mathrm{Bal} 31$ digestion. The recognition sequences for restriction sites are underlined.

quences were aligned by using the CLUSTAL multiple alignment software (14).

Nucleotide sequence accession number. The sequence of the $s s i$ site for $2 \mathrm{mDa}$ is from the GenBank-EMBL DNA sequence data base (accession no. M25995).

\section{RESULTS}

Deletion analysis of the SSO of pBAA1. The SSO of the plasmid pBAA1 is located on a 271-bp PstI-XmaIII fragment (Fig. 1). There are three dyad elements within this sequence with the potential to form three stem-loop structures (7). To delimit and further analyze this origin, plasmid pRP22 $\Delta$ Pst I was separately linearized with PstI and XmaIII, and each was progressively deleted by using $\mathrm{Bal} 31$. The endpoints of each deletion were determined by sequencing and are indicated by arrowheads in Fig. 1. Deleted plasmids were tested for SSO activity by estimation of the amount of accumulated single-stranded plasmid DNA. The extent of each deletion is shown in schematic form in Fig. 2, and the level to which single-stranded plasmid accumulated in cells containing these constructs is shown in Fig. 3. Deletion of the sequence between the PstI site and P15 (Fig. 1) has no detectable effect on SSO activity. Deletion from the PstI site to P6 (the deleted region extends to within 1 base of the stem of the predicted stem-loop I) leads to a low level of accumulation of single-stranded plasmid DNA in cells harboring this construct. In construct $\mathrm{pRP} / \mathrm{P} 7$, where half the sequence contributing to the formation of the proposed stem-loop $I$ is deleted, single-stranded plasmid DNA accumulates to the levels found with pBAA1 derivatives which have the entire SSO deleted. These data indicate that the formation of predicted stem-loop I and/or sequences within this predicted structure is essential for full activity of the SSO of pBAA1.

Deletions in the vicinity of the XmaIII site (e.g., pRP/X1), which do not affect the integrity of the proposed stem-loop 


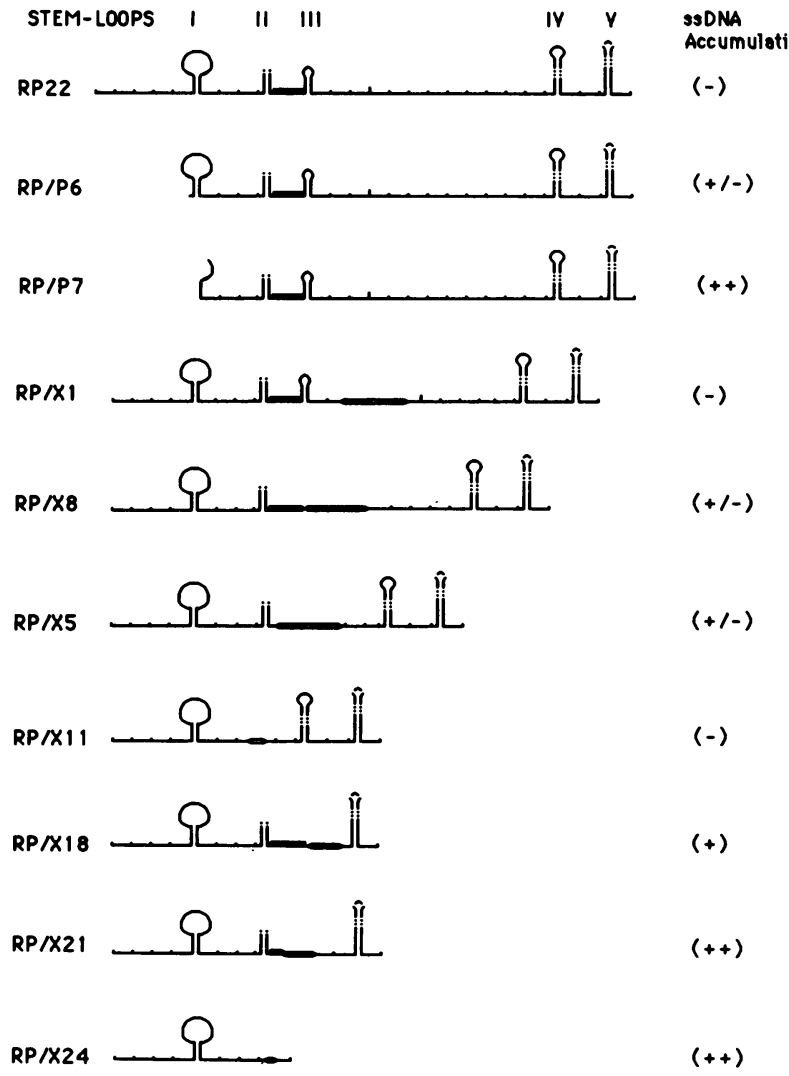

FIG. 2. Schematic representation of the SSO (the sequence of which is presented in Fig. 1) after deletion from the PstI (RP/P plasmids) and $\mathrm{XmaIII}$ (RP/X plasmid) sites. The structures are drawn to scale to indicate the relative positioning of the stem-loops (I through V) after deletion. Visual estimation of the amount of single-stranded plasmid DNA (ssDNA) which accumulates in cells is indicated as follows: $(-)$, not detectable; $(+/-)$, low level; $(+)$, intermediate level; $(++)$, the amount of single-stranded plasmid DNA which accumulates in cells harboring plasmid constructs which have the entire SSO structure deleted. The solid boxes located between predicted stem-loops II and III represent the conserved sequence motif 3 , which is partially ( $\mathrm{pRP} / \mathrm{X} 21)$ or completely (pRP/X11, X5, X24) deleted in some constructs. The open boxes represent linker sequences inserted into the plasmid after religation, the sizes of which are proportional to the number of linker units inserted.

structures, do not result in accumulation of single-stranded plasmid DNA. In contrast, levels of single-stranded plasmid DNA similar to those observed when the entire SSO is deleted were observed in cells harboring $\mathrm{pRP} / \mathrm{X} 21$ and $\mathrm{pRP} / \mathrm{X} 24$. These constructs have the proposed stem-loops III (pRP/X21) and II and III (pRP/X24) deleted. The SSO is not functional in either of these constructs, indicating a requirement for sequences and/or structures in the vicinity of these predicted stem-loops for origin activity. The nature of this requirement is revealed by comparison of the structures and results from $\mathrm{pRP} / \mathrm{X} 11$ and $\mathrm{pRP} / \mathrm{X} 24$. In both of these constructs, the deletion endpoints within the SSO are approximately the same; i.e., stem-loops II and III are deleted. In $\mathrm{pRP} / \mathrm{X} 24$, the predicted stem-loops IV and $\mathrm{V}$ are deleted, whereas in $\mathrm{pRP} / \mathrm{X} 11$ these two stem-loops are intact and are juxtaposed to stem-loop I. Cells harboring pRP/X24 accumulate large quantities of single-stranded plasmid DNA, whereas those harboring pRP/X11 do not, indicating that the

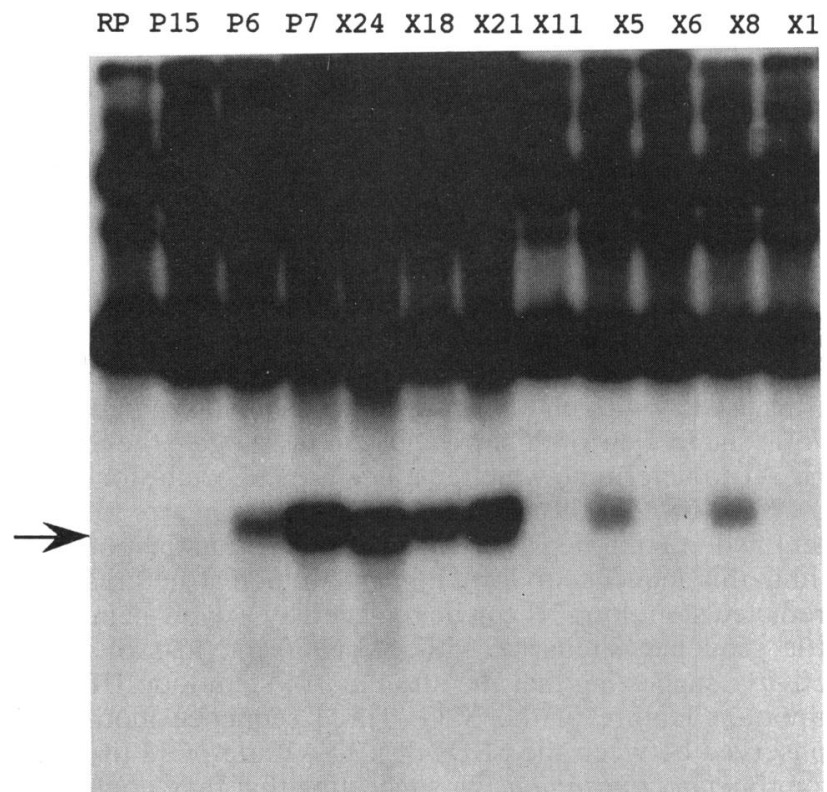

FIG. 3. Estimation of single-stranded plasmid DNA accumulation in cells harboring the deletion constructs. Whole-cell lysates were separated on agarose gels, denatured, transferred to nylon filters, and probed with the intact plasmid. Duplicate samples were similarly treated but transferred to filters without denaturation; only single-stranded plasmid will bind under these conditions. Only the bands opposite the arrow were visible on autoradiograms of nondenatured gels, indicating that these are single-stranded plasmid DNA species (data not shown). RP, plasmid pRP22 containing the intact SSO; P and X, deletion constructs from the PstI and XmaIII sites, respectively.

SSO is active in the latter construct. The predicted stemloops IV and V are not essential for activity of the intact SSO (7), and there is little sequence similarity between stem-loop III and stem-loops IV and V. This suggests that the predicted structure, rather than the sequence, of the stem-loop IV-V region is the feature responsible for the restoration of SSO activity observed in $\mathrm{pRP} / \mathrm{X} 11$. If stem-loop II forms as predicted, there are 48 nucleotides between the stems of stem-loops I and III in the intact origin and 55 nucleotides between the stems of stem-loops I and IV in pRP/X11. In constructs $\mathrm{pRP} / \mathrm{X} 5$ and $\mathrm{pRP} / \mathrm{X} 8$, the predicted stem-loops IV and $\mathrm{V}$ are located at a greater distance from stem-loop I than in $\mathrm{pRP} / \mathrm{X} 11$ and have reduced SSO activity. These data suggest that the location of a non-sequence-specific stemloop structure approximately 48 to 55 bases from stem-loop I is a feature which contributes to the activity of the SSO.

Identification of sequences within the SSO which are important for activity. In order to identify sequences within the SSO of pBAA1 which contribute to its activity, the GenBank data base was searched for plasmids containing homologous sequences. The plasmid pGI 2 (isolated from $B$. thuringiensis [19]) contains a segment of DNA, the sequence of which is $62 \%$ identical to the SSO of pBAA1 (Fig. 4). Comparison of these two sequences reveals a number of interesting features. This region of pGI2 has the potential to form three stem-loops with structures very similar to those of the SSO of pBAA1. The sizes of the loops in stem-loop I are 29 bases in pBAA1 and 59 bases in pGI2. The extra 30 bases in pGI2 are positioned between two regions of the loop where the sequence is conserved between the two plasmids (Fig. 4). 


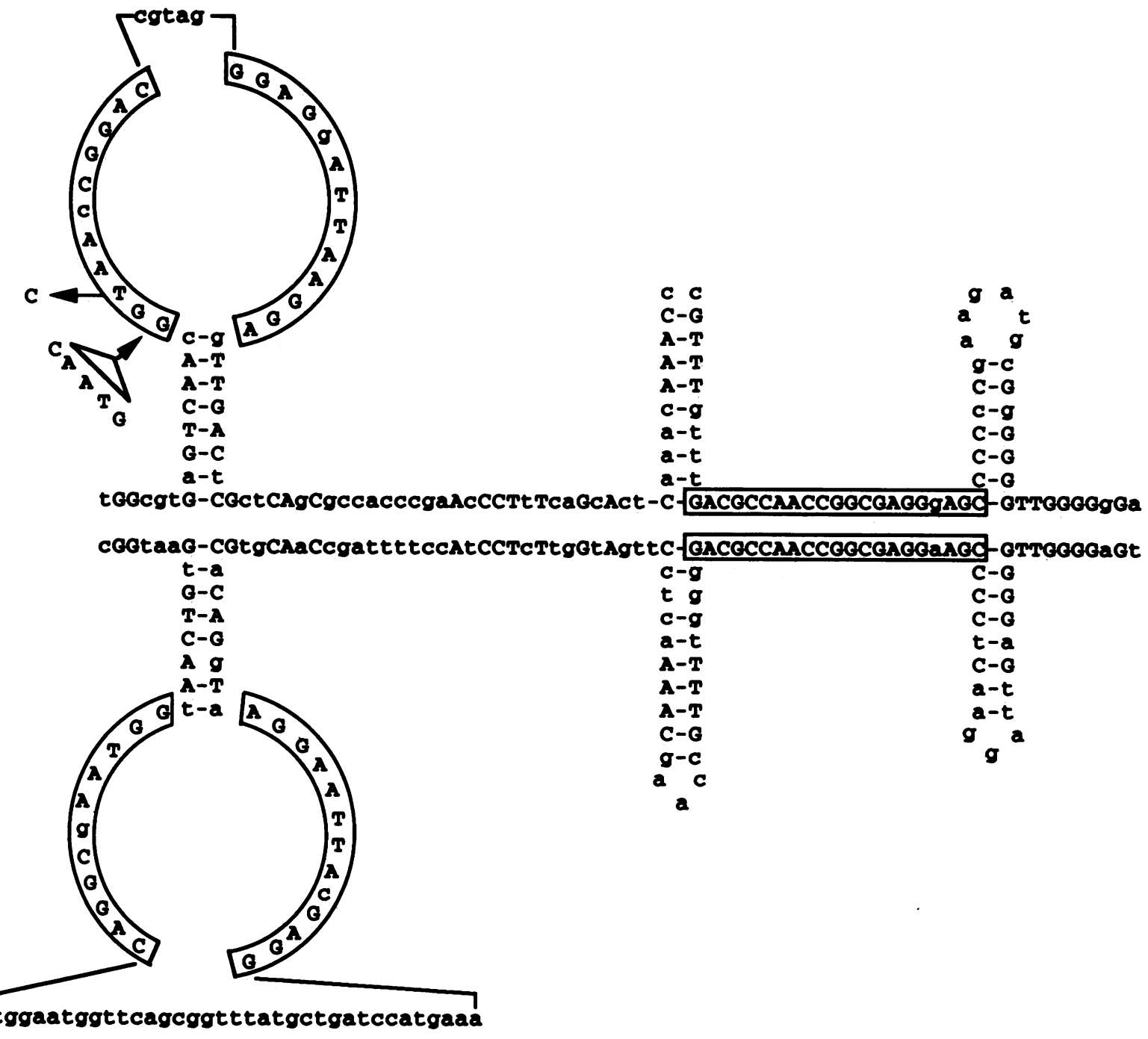

FIG. 4. Comparison between the sequence and predicted structure of the pBAA1 SSO (top sequence) and those of a homologous fragment from the plasmid pGI2 (bottom sequence). These fragments are $62 \%$ identical at the sequence level and are highly conserved with regard to the potential for secondary structure formation. Each sequence has the potential to form three stem-loop structures (stem-loops I, II, and III, left to right). Bases conserved between the two sequences are indicated by uppercase letters, and those not conserved are indicated by lowercase letters. Three sequence motifs (boxed sequences) are conserved between the two sequences: motifs 1 and 2, located in the left- and right-hand sides of stem-loop I, respectively, and motif 3, located between stem-loops II and III. Base pairing within the stems of the stem-loop structures is indicated by G-C. The positions of the $\mathrm{T} \rightarrow \mathrm{C}$ transition and the 5-bp insertion are indicated.

The importance of the conserved 11-base sequence GG TAA(G/C)CGGAC (motif 1, boxed in Fig. 4) was verified experimentally. There is a BstEII site within this sequence in pBAA1 which was used to generate a single base $\mathrm{T} \rightarrow \mathrm{C}$ transition and a 5-bp insertion (Fig. 4). Both of these mutations result in cellular accumulation of low levels of single-stranded plasmid DNA (data not shown), confirming the importance of this conserved sequence for SSO activity. There are 30 and 31 bases between the predicted stem-loops I and II of pBAA1 and pGI2, respectively, and 19 bases between the predicted stem-loops II and III of both plasmids, indicating that the relative positioning of these structures is critical for SSO activity. There is little similarity in the sequences located between stem-loops I and II of pBAA1 and pGI2. However, the sequences between the predicted stem-loops II and III of both plasmids are $95 \%$ identical, indicating that this sequence is important for the functioning of the SSO. This sequence (termed motif 3, boxed in Fig. 4) was used to search the GenBank data base for similar sequences. It was found to be conserved among a group of $10 \mathrm{ssi}$ sites, sequences shown to be involved in lagging-strand synthesis of a wide range of plasmids isolated from gram-negative bacteria (Fig. 5). Nine bases of the decamer GCGAGGAAGC are conserved between motif 3 and the ssi sites. However, 18 of 21 bases are identical among motif 3, ssiF from F, and an ssi site from p307. Conservation of the sequence and positioning of motif 3 in the SSOs of pBAA1 and pGI2 and the similarity with sequences from ssi sites isolated from gram-negative plasmids suggest that it contributes to origin activity. However, evidence from the deletion analysis with regard to the importance of this sequence in origin activity is conflicting. Motif 3 has been deleted from the origin construct in $\mathrm{pRP} / \mathrm{X} 11$, a construct which does not lead to cellular accu- 


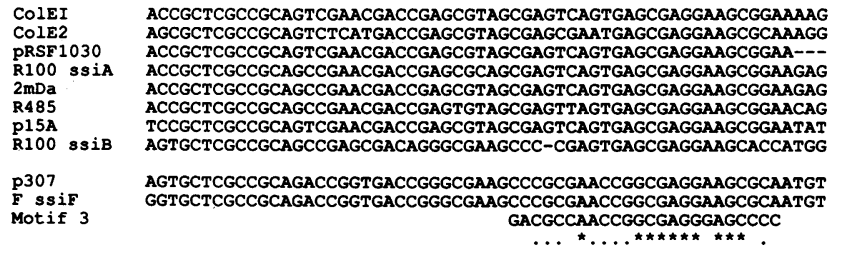

FIG. 5. Comparison between sequence motif 3 , conserved in the pBAA1 and pGI2 SSOs, and the sequences of ssi sites identified on plasmids isolated from $E$. coli. These $s s i$ sites are capable of acting as origins of complementary strand synthesis in an M13 phage derivative which has had its own minus origin deleted $(21,22)$. Bases conserved between motif 3 and all the ssi sites are indicated by a star, and those only conserved between motif 3 and ssi sites from p307 and $s s i F(F)$ are indicated by a dot. The sequences of the $s s i$ sites are from the following references: ssi $\mathrm{A}(\mathrm{ColE} 1)$, ssi $\mathrm{A}(\mathrm{ColE} 2)$, and $s s i \mathrm{~F}(\mathrm{~F}), 22 ; s s i \mathrm{~A}(\mathrm{R} 100)$ and $s s i \mathrm{~B}(\mathrm{R} 100), 24 ; 2 \mathrm{mDa}$, Materials and Methods; R485, 32; p15A, 31; and p307, 30.

mulation of single-stranded plasmid DNA, indicating that it is not essential for origin activity. Single-stranded plasmid DNA accumulates in cells harboring $\mathrm{pRP} / \mathrm{X} 8$ and $\mathrm{pRP} / \mathrm{X} 5$ to very similar levels, even though motif 3 is present in $\mathrm{pRP} / \mathrm{X} 8$ but is absent from $\mathrm{pRP} / \mathrm{X} 5$. The plasmids $\mathrm{pRP} / \mathrm{X} 18$ and $\mathrm{pRP} / \mathrm{X} 21$ are very similar, differing only in that motif 3 is present in $\mathrm{pRP} / \mathrm{X} 18$ but is substantially deleted from $\mathrm{pRP} /$ $\mathrm{X} 21$. Our results show that cells harboring $\mathrm{pRP} / \mathrm{X} 18$ accumulate less single-stranded plasmid DNA than cells harboring $\mathrm{pRP} / \mathrm{X} 21$, suggesting that motif 3 does contribute to origin activity. It is evident that the exact contribution of this motif to the activity of the SSO requires further investigation. Likewise, the role of motif 2 (boxed in Fig. 4), which is conserved between the SSOs of pBAA1 and pGI2 but is not found in any of the groups of ssi sites, remains to be elucidated.

Initiation of DNA synthesis at the SSO of pBAA1 is RNA polymerase dependent. The RNA primer used in conversion of the viral to the replicative form of the single-stranded phages is synthesized by DNA primase ( $\phi$ X174 and G4) and RNA polymerase (M13). To determine which of these enzymes synthesizes the primer at the SSO of pBAA1, cells harboring plasmids pRP22 (SSO $\left.{ }^{+}\right)$and pRP/X24 (SSO $\left.{ }^{-}\right)$ were grown in the presence and absence of rifampin, to which only RNA polymerase is sensitive. Accumulation of single-stranded plasmid replication intermediates in cells upon addition of rifampin would indicate that RNA polymerase is utilized to synthesize the primer. Figure 6 shows that single-stranded plasmid DNA accumulates in cells harboring pRP22 upon addition of rifampin, with maximal levels observed within $10 \mathrm{~min}$ of the start of the experiment. Levels declined during the remainder of the experiment. In cells harboring pRP/X24 (which has a nonfunctional, partially deleted SSO), single-stranded plasmid DNA is present before drug addition and levels do not increase in response to rifampin addition. However, like pRP22, levels decreased during the remainder of the experiment. These data indicate that RNA polymerase is involved in conversion of singlestranded plasmid to the double-stranded form. The reason for the decline in levels of single-stranded plasmid DNA during the experiment is unknown.

Host range of the pBAA1 SSO. pUB110 is unique among plasmids isolated from $S$. aureus in that its SSO functions both in its natural host and in $B$. subtilis (6). To investigate the functioning of the SSO of pBAAl in $S$. aureus, a HindIII-NaeI fragment of pBAA1 containing this origin was

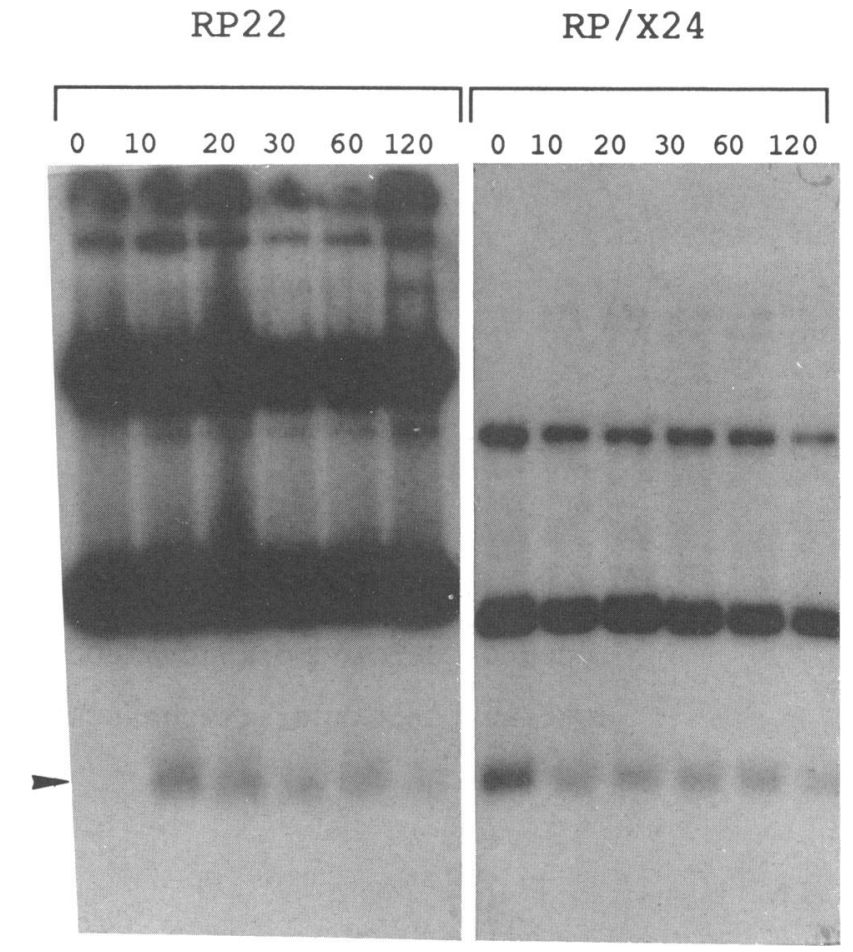

FIG. 6. Time course of single-stranded plasmid DNA accumulation in cells harboring plasmids pRP22 and pRP/X24 after addition of rifampin. Rifampin was added to cells to a final concentration of 100 $\mu \mathrm{g} / \mathrm{ml}$, and aliquots of cells were harvested before (time 0 ) and at 10 , $20,30,60$, and 120 min after drug addition. Single-stranded plasmid DNA accumulation was estimated as outlined in Materials and Methods. The autoradiograms shown are from gels denatured before transfer to nylon filters. A duplicate gel was transferred to nylon filters without denaturation; only the bands opposite the arrow were visible on this autoradiogram, indicating that these bands represent single-stranded plasmid DNA.

cloned in both orientations into a derivative of $\mathrm{pC} 194$ which has had its own SSO deleted. The criteria used to assess the SSO activity of this construct in $S$. aureus were (i) that the SSO has the ability to convert single-stranded plasmid DNA to the double-stranded form and (ii) that the SSO only functions in one orientation with respect to the doublestranded origin. Figure 7 (B and D) demonstrates that plasmids containing the SSO in the nonfunctional orientation accumulate large quantities of single-stranded plasmid DNA, whereas those with the origin in the functional orientation accumulate very low levels of single-stranded plasmid DNA (Fig. 7A and C). The orientation in which the SSO is functional in pC194 is the same with respect to the doublestranded origin as the orientation in which it is functional in pBAA1. Thus, the SSO of pBAA1 functions in both $B$. subtilis and $S$. aureus.

\section{DISCUSSION}

In this study, the features which contribute to the activity of the SSO of pBAAl were investigated. The SSO is contained on a fragment of DNA greater than 116 but less than 191 bases in size. Deletion of this sequence results in accumulation of single-stranded plasmid replication intermediates and in segregational instability of the plasmid (this study [7]). In this paper, evidence is presented which reveals 


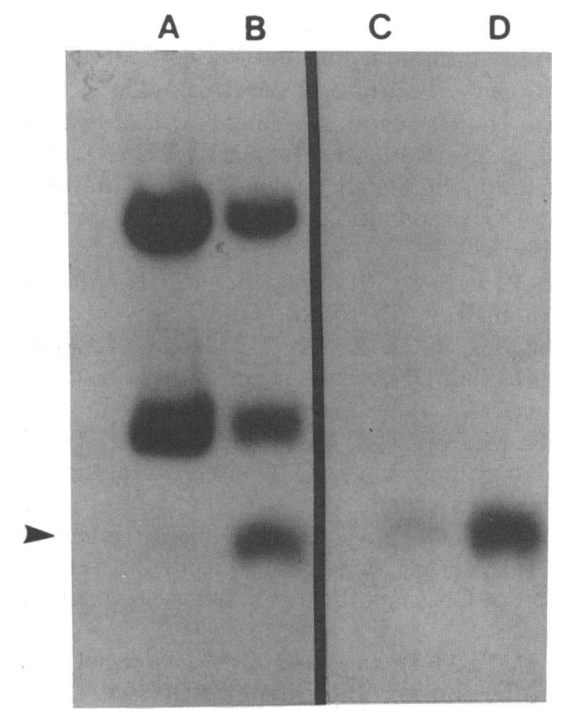

FIG. 7. The SSO of pBAAl, cloned in both orientations into a derivative of pC194 which had had its own SSO deleted, and transformed into $S$. aureus. Single-stranded plasmid DNA was visualized as outlined in Materials and Methods. Both the functional orientation (A and C) of the SSO in pC194 (i.e., the same orientation with respect to the double-stranded origin as that found in pBAA1) and nonfunctional orientation (B and D) are shown. Panels A and B were denatured and panels $C$ and $D$ were not denatured prior to transfer to nylon filters.

some of the features within this fragment which are important for SSO activity. A comparison of the sequences of the pBAA1 SSO and a homologous fragment from the plasmid pGI2 (isolated from $B$. thuringiensis) is informative with regard to structures and sequences which contribute to origin activity. These fragments from pBAA1 and pGI2 are $62 \%$ identical at the DNA sequence level. In addition, the structure of the predicted stem-loops and their relative positioning are highly conserved, suggesting that these features are important for origin activity. The importance of stem-loop structures within the SSO of pBAA1 is supported by the deletion analysis of the SSO-containing fragment. Deletion of sequences within the predicted stem-loop I structure leads to the abolition of origin activity, indicating that the predicted structure and/or sequences within this structure are important for origin activity. While it is not possible from these data to evaluate the separate contributions of the structure and sequence of stem-loop I to origin activity, the observed conservation of these features between pBAA1 and pGI 2 suggests that both are important.

Deletion of predicted stem-loop III (e.g., pRP/X21) leads to a reduction in origin activity. Replacement of stem-loop III with heterologous sequences capable of forming a stemloop structure (e.g., stem-loop IV in pRP/X11) leads to a restoration of SSO activity. We conclude that a non-sequence-specific stem-loop structure located at the position of stem-loop III is required for full origin activity. These observations are consistent with the fact that the potential to form secondary structure is a feature characteristic of all identified plasmid and phage SSOs. The minus origin of the G4 single-stranded phage, for example, has the potential to form three stem-loop structures. A systematic mutation analysis of this minus origin reveals that these structures are important for minus origin activity $(11,12,17,27)$.
A comparison of the sequences of the homologous fragments from pBAA1 and pGI2 reveals three motifs whose sequence and positioning are conserved. Two of these motifs (motifs 1 and 2) are located within the loop of predicted stem-loop I, while motif 3 is located between the predicted stem-loops II and III. Mutational analysis supports the conclusion that motif 1 contributes to origin activity. A single $\mathrm{T} \rightarrow \mathrm{C}$ transition or a 5-bp insertion within this sequence leads to attenuation of SSO activity. Both motif 1 and motif 3 are found in other sequences associated with lagging-strand synthesis. Motif 1 [GTAA(G/C)CGGAC] is found in pas $\mathrm{BH}$ of $\mathrm{pBR} 322$. Binding of PriA $\left(\mathrm{n}^{\prime}\right)$ to pas $\mathrm{BH}$ results in enhanced methylation of the underlined $G$ residue and protects the underlined $\mathrm{C}$ residue from attack by pancreatic DNase (8). Mutation of the underlined $C$ residue to an $\mathrm{A}$ residue affects the ability of pas $\mathrm{BH}$ to act as an effector of the ATPase activity of PriA. Motif 3 is a 21-bp sequence located between predicted stem-loops II and III. Nine bases of the decamer $\mathrm{GC}^{2} \mathrm{G}^{3} \mathrm{AGG} \mathrm{GAG}^{6} \mathrm{C}$ located within motif 3 are found in a group of ssi sites identified in $E$. coli plasmids. The importance of this sequence in initiation of laggingstrand synthesis at ssi sites is revealed by mutational analysis. Single base substitutions (changing the underlined bases to A residues) result in a decrease in the ability of the ssi site to activate the ATPase activity of PriA. Multiple base substitutions (changing $C^{2} G^{6} G^{9}$ or $G^{3} G^{9}$ to A residues) result in ssi sites which are not active with PriA (21). Thus, sequences associated with motifs 1 and 3 have been shown to contribute to PriA activation. PriA is the first protein to bind to DNA in the formation of the primosome. This suggests that lagging-strand synthesis in pBAA1 initiates at the SSO by a $\phi \mathrm{X} 174$ primosome-type mechanism. However, two pieces of evidence suggest that the mechanism whereby the pBAA1 SSO functions does not conform exactly to the $\phi X 174-$ type mechanism. Motif 3 is entirely deleted from $\mathrm{pRP} / \mathrm{X} 11$, a construct which has a functional SSO, indicating that this sequence is not essential for origin activity. In addition, the classic $\phi X 174$-type conversion mechanism uses DNA primase to synthesize the RNA primer, whereas our data indicate that RNA polymerase is utilized by the pBAA1 SSO. Thus, the pBAA1 SSO, although sharing sequence motifs with $\phi X 174$-type lagging-strand initiation sites, may function in $B$. subtilis by a different mechanism. While this analysis has revealed some of the features which contribute to the activity of the SSO of pBAA1, it is clear that determination of the precise mechanism by which laggingstrand synthesis is initiated requires further investigation.

\section{ACKNOWLEDGMENTS}

We thank Sandy Gruss for the pC194 $\Delta \mathrm{MO}$ construct and for assistance with $S$. aureus transformations.

This work was supported by grants from the European Commission Biotechnology Action Programme BAP-0038-IRL and BRIDGE Programme BIOT-CT-91-0268.

\section{REFERENCES}

1. Anagnostopoulos, C., and J. Spizizen. 1961. Requirements for transformation in Bacillus subtilis. J. Bacteriol. 81:741-746.

2. Arai, K., and A. Kornberg. 1981. Unique primed start of phage $\phi X 174$ DNA replication and mobility of the primosome in a direction opposite chain synthesis. Proc. Natl. Acad. Sci. USA 78:69-73.

3. Arai, K., R. L. Low, and A. Kornberg. 1981. Movement and site selection for priming by the primosome in phage $\phi \times 174$ replication. Proc. Natl. Acad. Sci. USA 78:707-711.

4. Baas, P. D. 1985. DNA replication of single-stranded Escherichia coli DNA phages. Biochim. Biophys. Acta 825:111-139. 
5. Baas, P. D., and H. S. Jancz. 1988. Single-stranded DNA phage origins. Curr. Top. Microbiol. Immunol. 136:31-70.

6. Boe, L., M.-F. Gros, H. te Riele, S. D. Ehrlich, and A. Gruss. 1989. Replication origins of single-stranded DNA plasmid pUB110. J. Bacteriol. 171:3366-3372.

7. Devine, K. M., S. T. Hogan, D. G. Higgins, and D. J. McConnell. 1989. Replication and segregational stability of Bacillus plasmid pBAA1. J. Bacteriol. 171:1166-1172.

8. Greenbaum, J. H., and K. J. Marians. 1984. The interaction of $E$. coli replication factor $\mathrm{Y}$ with complementary strand origins of DNA replication. J. Biol. Chem. 259:2594-2601.

9. Gruss, A., and S. D. Ehrlich. 1989. The family of highly interrelated single-stranded DNA plasmids. Microbiol. Rev. 53:231-241.

10. Gruss, A., H. F. Ross, and R. P. Novick. 1987. Functional analysis of a palindromic sequence required for normal replication of several staphylococcal plasmids. Proc. Natl. Acad. Sci. USA 84:2165-2169.

11. Hiasa, H., H. Sakai, K. Tanaka, Y. Honda, T. Komano, and G. N. Godson. 1989. Mutational analysis of the primer RNA template region in the replication origin (ori ${ }_{c}$ ) of bacteriophage G4: priming signal recognition by $E$. coli primase. Gene 84:9-16.

12. Hiasa, H., K. Yanaka, H. Sakai, K. Yoshida, Y. Honda, T. Komano, and G. N. Godson. 1989. Distinct functional contributions of three potential secondary structures in the phage G4 origin of complementary DNA strand synthesis. Gene 84:17-22.

13. Higgins, D. G., and M. Gouy. 1987. Interfacing similarity search software with sequence retrieval system ACNUC. Comput. Appl. Biosci. 3:239-241.

14. Higgins, D. G., and P. M. Sharp. 1989. Fast and sensitive multiple sequence alignments on a microcomputer. Comput. Appl. Biosci. 5:151-153.

15. Kim, M. H., J. C. Hines, and D. S. Ray. 1981. Viable deletions of the M13 complementary strand origin. Proc. Natl. Acad. Sci. USA 78:6784-6788.

16. Kornberg, A. 1980. DNA replication. W. H. Freeman and Company, San Francisco.

17. Lambert, P. F., E. Kawashima, and W. S. Reznikoff. 1987 Secondary structure at the phage G4 origin of complementarystrand DNA synthesis: in vivo requirements. Gene 53:257-264.

18. Lee, M. S., and K. J. Marians. 1989. The E. coli primosome can translocate actively in either direction along a DNA strand. J. Biol. Chem. 264:14531-14542.

19. Mahillon, J., and J. Seurinck. 1988. Complete nucleotide sequence of pGI2, a Bacillus thuringiensis plasmid containing Tn4430. Nucleic Acids Res. 16:11827.

20. Masai, H., N. Nomura, and K. Arai. 1990. The ABC primosome: a novel priming system employing dnaA, dnaB, dnaC and primase on a hairpin containing a $\operatorname{dna} A$ box sequence. J. Biol. Chem. 265:15134-15144.

21. Masai, H., N. Nomura, Y. Kubota, and K. Arai. 1990. Roles of $\phi X 174$ type primosome- and G4-type primase-dependent primings in initiation of lagging and leading strand syntheses of DNA replication. J. Biol. Chem. 265:15124-15133.

22. Nomura, N., H. Masai, M. Inuzuka, C. Miyazaki, E. Ohtsubo, H. Itoh, S. Sasamoto, M. Matsui, R. Ishizaki, and K. Arai. 1991. Identification of eleven single-strand initiation sequences for priming DNA replication in F, R6K, R100 and ColEII plasmids. Gene 108:15-22.

23. Novick, R. P. 1989. Staphylococcal plasmids and their replication. Annu. Rev. Microbiol. 43:537-565.

24. Ohtsubo, H., T. B. Ryder, Y. Maeda, K. Armstrong, and E. Ohtsubo. 1986. DNA replication of the resistance plasmid R100 and its control. Adv. Biophys. 21:115-133.

25. O'Reilly, M., and T. J. Foster (ed.). 1988. Immunochemical and molecular genetic analysis of bacterial pathogens, p. 199-207. Elsevier, Amsterdam.

26. Ray, D. S., J. C. Hines, M. H. Kim, R. Imber, and N. Nomura. 1982. M13 vectors for selective cloning of sequences specifying initiation of DNA synthesis on single-stranded templates. Gene 18:231-238.

27. Sakai, H., T. Komano, and G. N. Godson. 1987. Replication origin (ori $i_{c}$ ) on the complementary DNA strand of $E$. coli phage G4: biological properties of mutants. Gene 53:265-273.

28. Sambrook, J., E. F. Fritsch, and T. Maniatis. 1989. Molecular cloning: a laboratory manual. Cold Spring Harbor Laboratory Press, Cold Spring Harbor, N.Y.

29. Sanger, F., S. Nicklen, and A. R. Coulson. 1977. DNA sequencing with chain-terminating inhibitors. Proc. Natl. Acad. Sci. USA 74:5463-5467.

30. Saul, D., A. J. Spiers, J. McAnulty, M. G. Gibbs, P. L. Bergquist, and D. F. Hill. 1989. Nucleotide sequence and replication characteristics of RepFIB: a basic replicon of IncF plasmids. J. Bacteriol. 171:2697-2707.

31. Selzer, G., T. Som, H. Itoh, and J. H. Tomizawa. 1983. The origin of replication of plasmid p15A and comparative studies on the nucleotide sequence around the origin of related plasmids. Cell 32:119-129.

32. Stalker, D. M., and D. R. Helinski. 1985. DNA segments of the IncX plasmid R485 determining replication and incompatibility with plasmid R6K. Plasmid 14:245-254.

33. Stuitje, A. R., P. J. Weisbeek, and M. Meijer. 1984. Initiation signals for complementary strand DNA synthesis in the region of the replication origin of the $E$. coli chromosome. Nucleic Acids Res. 12:3321-3332.

34. te Riele, H., B. Michel, and S. D. Ehrlich. 1986. Single-stranded plasmid DNA in Bacillus subtilis and Staphylococcus aureus. Proc. Natl. Acad. Sci. USA 83:2541-2545.

35. Viret, J.-F., and J. Alonso. 1988. A DNA sequence outside the pUB110 minimal replicon is required for normal replication in Bacillus subtilis. Nucleic Acids Res. 16:4389-4406. 